\title{
Scleroderma and obstructive sleep apnea: a consideration of immunological aspects and the role of fibrosis
}

\author{
Ellen M. S. Xerfan ${ }^{1,2} \cdot$ Anamaria S. Facina $^{2}$ (D) - Jane Tomimori ${ }^{1,2} \cdot$ Sandra D. Xavier $^{3} \cdot$ Sergio Tufik $^{3}$. \\ Monica L. Andersen ${ }^{3}$
}

Received: 10 October 2020 / Revised: 4 February 2021 / Accepted: 12 February 2021 / Published online: 29 April 2021

(C) The Author(s), under exclusive licence to Springer Nature Switzerland AG 2021

Sleep disorders are known to be related to autoimmune diseases due to the inflammatory and immunological imbalance induced by or aggravating poor sleep [1]. In this context, we are writing to discuss a relationship that needs to be more clearly understood, that between obstructive sleep apnea (OSA) - a widely prevalent sleep breathing disorder [2] and scleroderma - an autoimmune disease that can affect breathing through a number of mechanisms, including lung fibrosis. We highlight pathological and immunological features that both diseases might have in common, and which warrant further clarification.

Scleroderma is an autoimmune disease that affects collagen and can present as a restricted dermatological condition, characterized by thickening of the skin, or as a systemic syndrome that evolves with a more severe clinical outcome. This systemic condition can be accompanied by significant collagen fibrosis that affects several organs beyond the skin, such as the esophagus, heart, and lungs, and carries with it great potential for health complications [1, 3], including impaired breathing that can worsen a pre-existing OSA.

Autoimmune and inflammatory diseases may lead to the impairment of sleep quality and a decrease in quality of life as a whole. In the case of scleroderma, it can cause lung complications, which can be manifested by interstitial lung disease and pulmonary hypertension, as well as restrictive-ventilatory limitation and anatomic changes in the upper airways due to

Anamaria S. Facina

dermatologista@hotmail.com

1 Programa de Pós-Graduação em Medicina Translacional, Universidade Federal de São Paulo (UNIFESP), São Paulo, Brazil

2 Departamento de Dermatologia, Universidade Federal de São Paulo (UNIFESP), R. Estado de Israel, 192 - Vila Clementino, São Paulo 04022-000, Brazil

3 Departamento de Psicobiologia, Universidade Federal de São Paulo (UNIFESP), São Paulo, Brazil fibrosis [1] as described below. These changes can affect sleep quality and sleep breathing movements, and are particularly relevant with respect to OSA and its interaction with scleroderma. We aim to discuss possible interaction between both conditions induced by these anatomic features, as well as with the potential role of immunological properties related to fibrosis.

Obstructive sleep apnea is a highly prevalent sleep breathing disturbance that affects one-third of the population $[2,4]$. The major risk factors for OSA are age, male sex, and obesity [2]. The morbidity and the mortality of OSA are high, and may be greater when it occurs concomitantly with other respiratory diseases, as those manifested in pulmonary involvement by systemic scleroderma $[4,5]$. Obstructive sleep apnea is characterized by recurrent episodes of apneas and hypopneas associated with repetitive episodes of intermittent hypoxia caused by repetitive collapse of the upper airway during sleep [4]. Hypoxemia and repetitive awakenings result in an increase in the number of circulating catecholamines and sympathetic activation that may predispose individuals to the worsening of several clinical outcomes of this sleep disorder and its associated respiratory pathophysiology [6]. Hypercapnia is another gasometrical disturbance that may be manifested in OSA, due to sleep breathing events and impaired $\mathrm{CO}_{2}$ wash out during sleep; this condition can also occur in some other respiratory system disorders that can coexist, such as obesity hypoventilation syndrome (OHS), chronic obstructive pulmonary disease (COPD), and the later stages of interstitial lung diseases (ILD) [6]. With respect to ILD that may also be manifested with hypoxemia [6], we highlight scleroderma, which could be another aggravating factor for OSA, worsening the gasometrical findings and the severity of these deleterious conditions, especially if they are concomitant.

One of the few studies to examine specifically the correlation between this autoimmune disease and sleep breathing disorders showed that one-third of the scleroderma patients 
had an associated sleep-disordered breathing [1]. It has previously been reported that OSA may present with higher severity and mortality when associated with other lung diseases. We highlight that OSA severity may be worsened by pulmonary fibrosis due to scleroderma. Restrictive pulmonary diseases, including those that occur in scleroderma are characterized by decreased lung volumes due to fibrosis, which can reduce upper airway stability and increase resistance by decreased traction $[4,5]$. These changes may facilitate upper airway collapse, mainly during stage REM sleep, when functional residual capacity is further reduced due to the inactivity of the intercostal muscles $[4,5]$, which demand a higher respiratory drive, impairing breathing and gas exchange during sleep.

A study revealed a high prevalence of OSA $(55.5 \%)$ in non-obese patients with scleroderma, with markedly impaired breathing during stage REM sleep [5]. It was concluded that there are different mechanisms for OSA other than obesity, such as the upper airway obstruction, and parenchymal and alveolar fibrosis associated with interstitial lung conditions [5]. Another study on this relationship found that OSA was detected in $44-72 \%$ of individuals with an interstitial lung disorder, which may aggravate its progression and related mortality [7], although these mechanisms are not yet clear. It is hypothesized that the intermittent hypoxia due to OSA may induce alveolar cell injury and cytokine release, which in turn may accelerate lung damage [8] and in parallel may be linked with immunological features that should be further clarified.

In addition to these pathological and mechanical reasons that could explain how fibrosis might induce greater OSA symptoms, we also call attention to current evidence that suggests that these diseases have an immunological characteristic in common - the inflammasome. This is a multiprotein intracellular complex that detects pathogenic microorganisms and sterile stressors, and that activates highly pro-inflammatory cytokines. Regarding the cytokines that may be activated by the inflammasome, we call particular attention to the interleukin (IL)-1 family [3], which includes members related to chronic lung diseases and fibrosis $[3,9]$. Several cytokines of this group seem to be related to fibrosis with some being known to promote a fibrotic response through fibroblast cells, such as IL-1, IL-18, and IL-33. These cytokines play an important role in skin and collagen regulation [3]. This may result in fibrosis in the lungs worsening symptoms of OSA.

Although the association between cytokines and the pulmonary effects of scleroderma has not been fully established, in another lung disorder, asthma, IL-33 has already been shown to be an important marker of fibrosis [3,9], and represents a promising therapeutic target [9]. We, therefore, propose a possible link between the profibrotic effects of the IL-1 family of inflammasomes in scleroderma, and the worsening of OSA. Research into the effects of increased levels of profibrotic cytokines, including mainly IL-33, may promote a better understanding of the immunological components that these two conditions possibly have in common and even guide therapeutic options.

On the other hand, studies have speculated that OSA plays a role in inflammation $[9,10]$. It has previously been reported that OSA may lead to greater systemic inflammation due to the intermittent hypoxia and the continued pauses in breathing during sleep [10]. Patients with OSA present with higher levels of the inflammatory marker IL-33, which may be released by the increased adipose tissue usually manifested in patients with OSA [9].

We suggest that in future research on this possible correlation between OSA and scleroderma fibrosis, the levels of the IL-1 family cytokines, especially IL-33, should be considered in the serum of patients to achieve a better understanding of this relationship. In addition, the hypothesis regarding the role of fibrosis in the severity of interstitial lung disorders, which includes the mechanical pathways and alveolar epithelium injury associated with gas exchange disturbance, should be raised in further research, as well as their immunological behavior. New findings on this possible interaction may provide a better understanding of the fibrosis pathways and biological markers that may be related to sleep disorders, especially OSA, and with pneumopathy severity. This effort may benefit the management of both of these diseases and guide new clinical protocols aiming to improve sleep and breathing.

Authors' contribution EMSX contributed to the conception, planning, acquisition of data, and original draft writing; ASF, JT, SDX, ST, and MLA contributed to the planning, acquisition of data, data analysis, intellectual contribution, and the final revision of the manuscript.

Funding Our studies are supported by the Associação Fundo de Incentivo à Pesquisa (AFIP). ST and MLA receive CNPq fellowships. EMSX receives a CAPES Post-Graduation grant. No sponsorship was received for the publication of this manuscript.

\section{Declarations}

All named authors meet the International Committee of Medical Journal Editors (ICMJE) criteria for authorship for this article, take responsibility for the integrity of the work as a whole, and have given their approval for this version to be published.

Conflict of interest The authors declare no conflict of interest.

\section{References}

1. Nokes BT, Raza HA, Cartin-Ceba R, Lyng PJ, Krahn LE, Wesselius L, Jokerst CE, Umar SB, Griffing WL, Neville MR, Malhotra A, Parish JM (2019) Individuals with scleroderma may have increased risk of sleep-disordered breathing. J Clin Sleep Med 15:1665-1669 
2. Tufik S, Santos-Silva R, Taddei JA, Bittencourt LRA (2010) Obstructive sleep apnea syndrome in the Sao Paulo epidemiologic sleep study. Sleep Medicine 11:441-446

3. Artlett CM (2018) The IL-1 family of cytokines. Do they have a role in scleroderma fibrosis? Immunol Lett 195:30-37

4. Lancaster LH, Mason WR, Parnell JA, Rice TW, Loyd JE, Milstone AP, Collard HR, Malow BA (2009) Obstructive sleep apnea is common in idiopathic pulmonary fibrosis. Chest 136 : 772-778

5. Pihtili A, Bingol Z, Kiyan E, Cuhadaroglu C, Issever H, Gulbaran Z (2013) Obstructive sleep apnea is common in patients with interstitial lung disease. Sleep Breath 17:1281-1288

6. Young IH, Bye PT (2011) Gas exchange in disease: asthma, chronic obstructive pulmonary disease, cystic fibrosis, and interstitial lung disease. Compr Physiol. 1:663-697

7. Myall KJ, West A, Kent BD (2019) Sleep and interstitial lung disease. Curr Opin Pulm Med 25:623-628
8. Ryan S, Taylor CT, McNicholas WT (2005) Selective activation of inflammatory pathways by intermittent hypoxia in obstructive sleep apnea syndrome. Circulation 112:2660-2667

9. Gabryelska A, Kuna P, Antczak A, Białasiewicz P, Panek M (2019) IL-33 Mediated inflammation in chronic respiratory diseases - understanding the role of the member of IL-1 superfamily. Front Immunol 10:692

10. Carpagnano GE, Spanevello A, Sabato R, Depalo A, Palladino GP, Bergantino L, Foschino Barbaro MP (2010) Systemic and airway inflammation in sleep apnea and obesity: the role of ICAM-1 and IL-8. Transl Res 155:35-43

Publisher's note Springer Nature remains neutral with regard to jurisdictional claims in published maps and institutional affiliations. 